\title{
A Comparative Study of Online Privacy Policies and Formats
}

\author{
Aleecia M. McDonald, ${ }^{1}$ Robert W. Reeder, ${ }^{2}$ Patrick Gage Kelley, ${ }^{1}$ \\ Lorrie Faith Cranor ${ }^{1}$ \\ 1 Carnegie Mellon, Pittsburgh, PA \\ 2 Microsoft, Redmond, WA
}

\begin{abstract}
Online privacy policies are difficult to understand. Most privacy policies require a college reading level and an ability to decode legalistic, confusing, or jargon-laden phrases. Privacy researchers and industry groups have devised several standardized privacy policy formats to address these issues and help people compare policies. We evaluated three formats in this paper: layered policies, which present a short form with standardized components in addition to a full policy; the Privacy Finder privacy report, which standardizes the text descriptions of privacy practices in a brief bulleted format; and conventional non-standardized human-readable policies. We contrasted six companies' policies, deliberately selected to span the range from unusually readable to challenging. Based on the results of our online study of 749 Internet users, we found participants were not able to reliably understand companies' privacy practices with any of the formats. Compared to natural language, participants were faster with standardized formats but at the expense of accuracy for layered policies. Privacy Finder formats supported accuracy more than natural language for harder questions. Improved readability scores did not translate to improved performance. All formats and policies were similarly disliked. We discuss our findings as well as public policy implications.
\end{abstract}

* Funded by NSF Cyber Trust grant CNS-0627513, Microsoft through the Carnegie Mellon Center for Computational Thinking, Army Research Office grant number DAAD19-02-1-0389 to Carnegie Mellon CyLab, and FCT through the CMU/Portugal Information and Communication Technologies Institute. Thanks to Robert McGuire and Keisha How for programming assistance. 


\section{Introduction}

The United States relies on a self-regulation approach to Internet privacy. There are some Internet privacy laws, for example the Children's Online Privacy Protection Act of 1998 (COPPA), which protects children's privacy [6], and the Gramm-Leach-Bliley Act (GLB), which applies to financial data [11]. But by and large the theory of Internet privacy hinges on two assumptions:

- Consumers will choose companies with acceptable privacy policies.

- Companies will not violate their privacy policies because the Federal Trade Commission (FTC) can bring action for unfair and deceptive practices.

In both cases privacy policies play a vital role in Internet privacy. Self-reports show three quarters of Internet users take active measures to protect their privacy, ranging from installing privacy protective technology to providing false information to web sites [1]. Yet only $26 \%$ read privacy policies during a recent study and readership outside of laboratory conditions is believed to be far lower [14]. To study the effectiveness of various approaches to improving the readability of privacy policies, we investigated the performance of three different formats for privacy policies and compared policies from six different companies.

In section two we describe related work and the formats we contrasted. We describe our methods in section three. We present accuracy and time to answer results in section four, and psychological acceptability results in section five. We discuss implications from these results and conclude in section six.

\section{Related Work}

Several studies frame willingness to read privacy policies as an economic proposition and conclude that asymmetric information is one reason why people find it not worth their time to read privacy policies $[28,1]$. Other studies show that privacy policies and financial disclosures require a college reading level to understand $[12,24,10,2]$. A study of ambiguities in privacy policies shows they contain language that downplays privacy issues [20]. The 2006 Kleimann report on GLB financial privacy notices found that subheadings and standard formats dramatically improved readability [22]. In response to these issues, privacy researchers and industry groups devised several standardized formats for privacy policies based on the expectation that standardized formats would improve comprehension. Our study is a comparative analysis to analyze how well standardized policies work in practice.

While not in the realm of privacy policies, Kay and Terry's research on open source license agreements includes testing multiple formats. Early work found modest improvements in likelihood to read well designed agreements but no improvement in retention of the material [15]. Tsai found when study participants searched for products to purchase and saw a single icon view that evaluated the privacy practices for each site, they were willing to pay a small premium for more privacy-protective sites $[27,8]$. On the other hand, translating an entire privacy 
policy into a grid that conveyed information by icons and colors did not improve comprehension [21]. Attempts at visualizing privacy are ongoing, including a set of icons modeled after Creative Commons [3]. This study, in contrast, examines three text-based formats as described below.

\subsection{Privacy Finder}

Privacy Finder (PF) is a privacy-enhanced front end to Yahoo! and Google search that was developed by AT\&T and refined at the Cylab Usable Privacy and Security (CUPS) Laboratory. Privacy Finder includes a privacy report that displays standardized text generated automatically from Platform for Privacy Preferences (P3P) policies. P3P is a standardized format for privacy policies, and is formally recommended by the World Wide Web Consortium (W3C) [29]. P3P policies are encoded in XML (eXtended Markup Language), which is computer readable and thus allows software tools to help people manage their privacy preferences.

Because Privacy Finder generates text from P3P tags, the Privacy Finder report avoids emotionally charged language and ensures uniform presentation. However, Privacy Finder reports allow a free-form text description of the highest level of policy statements. This can improve readability by providing context for readers, but also means that companies with identical practices may have different Privacy Finder reports.

\section{$2.2 \quad$ Layered Notices}

The law firm Hunton \& Williams popularized the notion of layered notices [25] which include a short one-screen overview with standardized headings which then links to the full natural language policy. Although the headings for the first layer are standardized the text within each section is free form.

By 2005, several large companies deployed layered policies including Microsoft (MSN), Procter \& Gamble, IBM, and JP Morgan [17]. European Union Information Commissioner Richard Thomas called for the use of layered policies in response to research showing nearly $75 \%$ of participants said they would read privacy policies if they were better designed [19]. Article 29 of European Union Directive created the "Working Party on the Protection of Individuals with regard to the processing of Personal Data," which issued guidance on how to create layered policies [4]. Privacy commissioners in EU countries supported layered policies. In Australia, the Privacy Commissioner released a layered policy for their own office, intending it "as a model for other agencies and organisations" $[26]$.

\subsection{Natural language}

Most privacy policies are in natural language format: companies explain their practices in prose. One noted disadvantage to current natural language policies is that companies can choose which information to present, which does not 
necessarily solve the problem of information asymmetry between companies and consumers. Further, companies use what have been termed "weasel words" - legalistic, ambiguous, or slanted phrases - to describe their practices [20]. Natural language policies are often long and require college-level reading skills. Furthermore, there are no standards for which information is disclosed, no standard place to find particular information, and data practices are not described using consistent language.

\section{Methods}

We conducted an online study from August to December 2008 in which we presented a privacy policy to participants and asked them to answer questions about it. We posted advertisements on craigslist and used personal networks to recruit participants. We offered a lottery for a chance to win one of several $\$ 75$ Amazon gift certificates as incentive for participating in the study.

We used a between subjects design and assigned each participant to one of 15 privacy policy representations. We used a between subjects design rather than within group design because in this context it is unrealistic to eliminate learning effects simply by reordering policies. Reading the questions could affect how participants read subsequent policies. It is also unrealistic to expect participants to spend more than 20 minutes completing an online survey. Questions remained constant over all conditions; only the policy differed.

\subsection{Study Conditions}

We contrasted six different companies' conventional natural language (NL) policies and their corresponding Privacy Finder privacy report format (PF) plus three layered policies. We refer to these companies as A through F. We analyzed 749 participants across 15 conditions, for an average of 50 participants per condition. The study conditions are listed in Table 1.

Table 1. Participants per Condition

\begin{tabular}{|l|c||c|c|c|}
\hline Company & Designation & NL & PF & Layered \\
\hline Disney & A & 41 & 50 & N/A \\
Microsoft & B & 47 & 46 & 52 \\
Nextag & C & 46 & 41 & N/A \\
IBM & D & 47 & 47 & 49 \\
Walmart & E & 52 & 51 & N/A \\
O'Reilly & F & 62 & 55 & 63 \\
\hline
\end{tabular}

We replaced all companies' names with "Acme" to avoid bias from brand effects. For natural language polices we used black text on white backgrounds 
regardless of the original graphic design. We left other formatting that might aide comprehension (for example, bulleted lists) intact.

Note that we did not study layered policies for companies A, C, and E. Of the six companies, only B and D had layered policies. We followed the directions from the Center for Information Policy Leadership [5] to create a third layered policy for company $\mathrm{F}$ as part of a prior study [21] and used it here to facilitate comparisons between studies.

As deployed in practice, Privacy Finder highlights the most important information at the top of the report and provides links to expand details. We discovered in earlier testing that people rarely expanded the Privacy Finder report. We were interested in testing how well people are able to use the information in the Privacy Finder report, not how well they are able to navigate the user interface, so in our research we presented all information in a single flat file.

We selected privacy policies from six popular websites that engage in ecommerce, and thus must collect a variety of personal information as part of their business. We chose what we believe to be a comparatively easy to read and a comparatively difficult to read policy with several typical policies. We selected policies guided by several measurements of readability summarized in Table 2. For each company, we noted the length of the natural language policy. We calculated the Flesch-Kincaid Reading Ease Score, which ranges from a low of 1 to a high of 100 based on syllable count and line lengths. High FleschKincaid scores are more readable than low scores. In general, experts suggest a score of at least $60-70$, which is considered easily understandable by 8 th and 9th graders [18]. Reader's Digest has a readability index in the mid 60s, Time is in the low 50s, and Harvard Law Review in the low 30s [13]. Note that while the policies we selected span a range from 32 to 46 , even the most readable policy is more challenging than is normally recommended for a general audience.

We calculated the percentage of sentences written in the passive voice, which is both more difficult for readers to understand and an indicator the company may not be comfortable taking full responsibility for their privacy practices. We counted the number of cross references within each policy; the more times readers are asked to refer to other parts of the document the more difficult it is to understand. Finally, we note that the standardized Privacy Finder format also has a range of lengths due to differing numbers of statements, how much information they collect, and how much text the policy authors elected to supply.

\subsection{Study Questions}

Study questions comprised several groups:

- Comprehension. Participants answered a series of multiple choice questions to determine how well they were able to understand the policy. These questions are realistic information retrieval tasks based on typical privacy concerns, and are similar to questions used in an earlier study by Cranor et al [7]. In the study, we conducted three rounds of pilot tests with over two dozen people to ensure the questions were well-worded and understandable. 
Table 2. Attributes of six companies' privacy policies

\begin{tabular}{|c|c|c|c|c|c|c|}
\hline Co. & NL Words & NL Pages & Flesch & \% Passive & Cross ref.s & PF Words \\
\hline A & 6329 & 13 & 31.8 & $11 \%$ & 27 & 880 \\
B & 3725 & 7 & 35.5 & $22 \%$ & 0 & 1964 \\
C & 2920 & 6 & 36.3 & $17 \%$ & 7 & 2011 \\
D & 2586 & 8 & 42.8 & $18 \%$ & 2 & 554 \\
E & 2550 & 8 & 44.9 & $11 \%$ & 0 & 1373 \\
F & 928 & 3 & 46.3 & $9 \%$ & 1 & 1843 \\
\hline
\end{tabular}

We randomized the order of these questions to mitigate learning effects and captured both accuracy and time to respond. We also included a warm-up task which we did not score.

- Psychological Acceptability. Saltzer and Schroeder coined the term psychological acceptability to convey that if people do not like a system they will not use it. They wrote, "It is essential that the human interface be designed for ease of use, so that users routinely and automatically apply the protection mechanisms correctly." [23] Participants answered subjective questions on a seven-point Likert scale.

- Demographics. We collected basic information like gender, educational attainment, and income so we could understand how closely our study population resembles Internet users as a whole.

We also measured the time it took for participants to answer each one of the comprehension questions. When not engaged in a research study, few people even skim privacy policies let alone read them to find answers to their concerns [15]. The times we measured do not reflect normal practices, but they do allow us to compare performance between formats, which is our goal.

\subsection{Research Questions}

Standardized formats were designed with care to help readers make sense of online privacy policies. With all of the resources invested in standardized policies we expected they would help people understand privacy policies. We held multiple hypotheses:

- Participants will have (a) higher accuracy scores, (b) shorter times to answer, and (c) greater psychological acceptability with both of the standardized formats than with their natural language counterparts.

- Participants will have (a) higher accuracy scores, (b) shorter times to answer, and (c) greater psychological acceptability with highly readable natural language than they will on natural language policies with low readability metrics.

Understanding these issues contributes to determining the most effective ways to present policies to end users. This is particularly relevant given Gramm- 
Leach-Bliley regulations on paper-based financial privacy policies; similar legislation could apply to online privacy policies in the future. The FTC's most recent report on behavioral advertising was described by the FTC Chairman Leibowitz as the last chance to make industry self-regulation work [9]. If we move away from industry self-regulated content, what should we do instead? Do any of the standardized approaches help enough to warrant considering regulation of policy formats?

\subsection{Analysis}

We performed a comparative analysis across all three formats (Natural Language, Privacy Finder, and Layered) and from all six companies to see if there were statistically significant differences in the mean scores for accuracy, time to completion, and psychological acceptability questions.

After we removed outliers ${ }^{3}$ we performed ANOVA analysis for both time data and psychological acceptability, which we recorded on a seven point Likert scale and treated as continuous variables. We performed all tests of statistical significance at the $\alpha=5 \%$ confidence level. For the sake of readability, all details of statistical significance tests are in the Appendix.

\section{Accuracy and Speed Results}

Accuracy scores are all reported as the percentage of people who answered the question correctly. ${ }^{4}$ As compared to natural language, we found that layered policies led to lower accuracy scores for topics not in the short layer. Privacy Finder

\footnotetext{
${ }^{3}$ We only included results from participants who completed all of the accuracy questions. Because this was an online study to enter a drawing for a gift certificate, a few people just "clicked through" answers without engaging with the material. We picked a fixed lower threshold of 1.5 seconds per question and removed participants entirely if they had two or more questions they answered in under 1.5 seconds ( 7 participants removed out of an original 756 for a total of 749.) For participants with only one time under 1.5 seconds, it is possible they accidently double-clicked once but answered other questions properly. We removed the time and accuracy data for just the affected question (3 question/time pairs out of 3000.) At the other extreme, sometimes people were diverted by other tasks while answering questions and we recorded unduly long times to answer. We discarded question times in excess of 2.5 times the mean for their condition along with their corresponding answers. This resulted in $N=723$ for cookies, 728 for opt out, 726 for share email, and 723 for the telemarketing questions.

${ }^{4}$ Interpreting results is complicated by potential confusion of how participants answered when answers are inferred. For example, we asked about opt out practices for policies where there is no opt out link. The straight-forward answer we envisioned is "No." However, participants may also have replied that the policy "Does Not Say," intending to convey the same information since there is no opt out link within the policy. Arguably, in that case the correct way to score responses is to combine the correct answer with "Does Not Say." We analyzed the combined percentage for each question and found in all but one case there was no difference in
} 
was indistinguishable from natural language until questions became harder, at which point Privacy Finder was slightly superior to natural language.

Accuracy spanned a wide range. An average of $91 \%$ of participants answered correctly when asked about cookies, $61 \%$ answered correctly about opt out links, $60 \%$ understood when their email address would be "shared" with a third party, and only $46 \%$ answered correctly regarding telemarketing. With only three possible answers, if participants guessed randomly we would expect $33 \%$ accuracy.

All other things being equal, lower times are better because they reflect participants were better able to comprehend the policy. Participants answered more quickly with both layered and Privacy Finder formats. Times to answer increased with question difficulty, with an average of 2.3 minutes to answer the question about cookies, 4.7 minutes to answer about opt out links, 5.3 minutes for email sharing, and 6.7 minutes for telemarketing.

\subsection{Cookies}

We asked: Does the Acme website use cookies?

Answer: Yes for all policies.

Most participants got the cookie question right (91\%). This was an easy question to answer because our question is phrased with the same term the policies use. All policies, in all formats, call out cookies use explicitly. For example, one policy has a heading of "Cookies and Other Computer Information" with a paragraph that begins: "When you visit Acme.com, you will be assigned a permanent 'cookie' (a small text file) to be stored on your computer's hard drive." There is no ambiguity. Even someone who has no idea what a cookie is, or what the implications for privacy are, can skim through any of the natural language policies to find the word "cookie" and answer correctly.

We found significant differences in accuracy for company and format. The six companies have a relatively small span between the worst performance (D, 82\%) and best performance (E, 96\%.) See Table 3 for a summary of results.

Layered policies gave participants a little more trouble $(78 \%)$ than other formats. Cookie information was under the heading "Personal Information" in F Layered $(80 \%$,) which may not be where

Table 3. Percentage correct and minutes to answer, cookies question.

\begin{tabular}{|l|c|c|}
\hline Policy & \% correct & Time \\
\hline A NL & $87 \%$ & 3.6 \\
A PF & $96 \%$ & 1.5 \\
\hline B NL & $96 \%$ & 2.0 \\
B PF & $98 \%$ & 1.6 \\
B Layered & $86 \%$ & 2.3 \\
\hline C NL & $93 \%$ & 2.4 \\
C PF & $98 \%$ & 3.5 \\
\hline D NL & $86 \%$ & 2.6 \\
D PF & $91 \%$ & 1.9 \\
D Layered & $69 \%$ & 2.2 \\
\hline E NL & $96 \%$ & 2.6 \\
E PF & $96 \%$ & 1.8 \\
\hline F NL & $100 \%$ & 2.3 \\
F PF & $94 \%$ & 2.7 \\
F Layered & $80 \%$ & 2.3 \\
\hline
\end{tabular}
people expected to look. In D Layered $(69 \%$,$) the$ policy mentions in passing that "You may also turn off cookies in your browser,"

the threshold for statistical significance. Further, the relative ranking of formats and companies remained stable. 
without explicitly saying they use cookies. People must deduce that information or go to the full policy for a direct statement that the site uses cookies. This highlights two results we will see again: first, when participants needed to think about an answer rather than just perform a search for information, accuracy dropped. Second, it appears few people ventured beyond the first page of the layered policies. Kay and Terry found similar issues with layered policies [15].

In another sign that this was an easy question for most participants, times to answer were shorter than the other questions (2.3 minutes.) We found no significance for time based on company but format was significant. Privacy Finder (2.1 minutes) and Layered (2.3 minutes) supported faster responses than Natural Language, but the Layered condition was also more likely to result in incorrect answers.

\subsection{Opt Out Link}

We asked: Does the company provide a link to a webform that allows you to remove yourself from Acme's email marketing list?

Answer: Yes for all policies except: B NL, D NL, D Layered, E NL, which are No. ${ }^{5}$

This question is a little more difficult than the question about cookies. Policies refer to this concept as "opting out." For example, company C's natural language policy phrases it as "To opt out of receiving all other Acme mailings after you have registered, click here or click the appropriate unsubscribe link contained within the email that you receive." Participants need to map the concept of removing themselves from an email marketing list to the technical jargon of opting out. However, this question is again fairly straight forward. Either there is an opt out link or there is not. See Table 4 for a summary of results.

We found significant differences for company and format. Natural language policy accuracy rates are dissimilar, with averages ranging from $93 \%$ (F) to $33 \%$ (A). Finding the opt out

Table 4. Percentage correct and minutes to answer for the opt out question.

\begin{tabular}{|l|c|c|}
\hline Policy & \% correct & Time \\
\hline A NL & $33 \%$ & 5.7 \\
A PF & $85 \%$ & 3.7 \\
\hline B NL & $33 \%$ & 9.3 \\
B PF & $91 \%$ & 4.6 \\
B Layered & $18 \%$ & 4.8 \\
\hline C NL & $80 \%$ & 3.2 \\
C PF & $73 \%$ & 5.1 \\
\hline D NL & $29 \%$ & 6.1 \\
D PF & $71 \%$ & 3.8 \\
D Layered & $19 \%$ & 5.5 \\
\hline E NL & $55 \%$ & 5.4 \\
E PF & $51 \%$ & 4.6 \\
\hline F NL & $93 \%$ & 3.4 \\
F PF & $79 \%$ & 3.7 \\
F Layered & $92 \%$ & 2.2 \\
\hline
\end{tabular}
link in the A NL policy was looking

\footnotetext{
${ }^{5}$ Answers are not the same across a given company because the companies elected to provide different information in different formats. P3P requires an opt out link, which is then included in Privacy Finder.
} 
for a needle in a haystack: there is one link halfway through the policy in the middle of a paragraph without any headings or other cues - and the policy runs to 13 pages when printed.

It would seem Privacy Finder should have consistent results across all six policies, since an opt out link is a standard part of Privacy Finder reports. However, companies with an opt out default have additional links for each category of opt out data. As a result, policies with opt out practices fared better, ranging from $85 \%$ correct (A $\mathrm{PF}$ ) with less privacy protective practices and many prominent opt out links, to $51 \%$ correct (E PF) which required opt out for all data collection and had only one opt out link. Interestingly, the F PF policy (79\%) has identical practices as E PF (51\%) yet different accuracy scores. The author of the F PF policy included an additional opt out link in the text at the very end of the policy, which is prime real estate for readers' attention. Policy authors choices affect outcomes, even within the PF standardized presentation.

Since there is no requirement to discuss opt out choices within the layered format, once again we see dissimilar results across a standardized format. B layered policy (18\%) required clicking the opt out link to see what it did, phrased as "For more information about our privacy practices, go to the full Acme Online Privacy Statement. Or use our Web form," with a link from "Web form" to the opt out page. In contrast, results were quite good with F layered (92\%), which contained the same opt out text as at the end of the F PF (79\%) policy.

We found significant differences in time to answer for company as well as format. We would expect longer times for longer policies since this is in many ways an information search task. Instead, time appears to be based on the underlying practices: policies without opt out links took longer. Since some of the policies with opt out links mentioned them at the end, it is unlikely the difference in times is based on reading through the entire policy to determine the absence of a link. Instead, participants likely re-read to satisfy themselves that they had not missed anything. Once again participants completed the task more quickly with layered (4.0 minutes) and Privacy Finder (4.2 minutes) than Natural Language (5.4 minutes,) but the wide variance and sometimes poor performance for standardized policies reduces the strength of this result.

Table 5. Percentage correct and minutes to answer for the email sharing question.

\begin{tabular}{|l|c|c|}
\hline Policy & \% correct & Time \\
\hline A NL & $76 \%$ & 3.2 \\
A PF & $53 \%$ & 5.4 \\
\hline B NL & $49 \%$ & 5.9 \\
B PF & $64 \%$ & 5.9 \\
B Layered & $52 \%$ & 4.8 \\
\hline C NL & $80 \%$ & 4.7 \\
C PF & $72 \%$ & 6.9 \\
\hline D NL & $67 \%$ & 4.6 \\
D PF & $78 \%$ & 4.0 \\
D Layered & $56 \%$ & 4.7 \\
\hline E NL & $53 \%$ & 6.9 \\
E PF & $44 \%$ & 6.2 \\
\hline F NL & $50 \%$ & 6.0 \\
F PF & $54 \%$ & 4.4 \\
F Layered & $62 \%$ & 5.0 \\
\hline
\end{tabular}




\subsection{Share Email}

We asked: Does this privacy policy allow Acme to share your email address with a company that might put you on their email marketing list (with or without your consent)?

Answer Yes for all policies except: companies E and F (all formats) which are No.

We tested the wording of this question in multiple pilot studies to ensure people understood it without asking something pejorative or jargon-laden like "will Acme sell your email address to spammers." This question requires participants to understand the question, read the policy carefully, and make inferences for most policies. For example, C NL reads: "We may provide your contact information and other personal data to trusted third parties to provide information on products and services that may be of interest to you." Participants need to understand that "contact information" includes email, that "trusted third parties" are companies other than Acme, and that "provide information on products and services" means marketing messages, in order to correctly answer "Yes." See Table 5 for a summary of results.

Overall accuracy was only $60 \%$. We found significant differences for company but not format. Times to answer averaged 5.3 minutes, which indicates people had a harder time completing this task. We found no significant results for time based on company or format.

As the answers to our questions become more nuanced we would expect the more readable policies to shine, yet that is not the case. Company A, with the hardest to read policy, had a higher accuracy score $(64 \%)$ than $\mathrm{F}(55 \%)$ with the most readable policy and there was no overall discernible pattern based on readability. Similarly, we would expect standardized policies to convey information better, especially the Privacy Finder format which avoids the emotion-rich wording of "trusted third parties" and "valuable offers," yet we did not find significant differences between formats. Privacy Finder summarizes "With whom this site may share your information" as "Companies that have privacy policies similar to this site's" which again requires participants to refer to a separate section to determine if the parent company may engage in email marketing.

\subsection{Telemarketing}

We asked: Does this privacy policy allow Acme to use your phone number for telemarketing?

Answer Yes for all policies except companies A, E and F (all formats) which are No.

Participants struggled with this question as shown in Table 6. Except in the Privacy Finder version where companies are required to provide information about their telemarketing practices, policies typically do not highlight telemarketing practices. The way to answer this question correctly was typically to read through the entire policy for all mentions of when the company collects phone numbers, then see what policies they have around that data. For example, B NL 
discloses telemarketing as: "You may also have the option of proactively making choices about the receipt of promotional e-mail, telephone calls, and postal mail from particular Acme sites or services." Sometimes policies were even more vague, for example D NL, "The information you provide to Acme on certain Acme Web sites may also be used by Acme and selected third parties for marketing purposes. Before we use it, however, we will offer you the opportunity to choose whether or not to have your information used in this way." Not only is telemarketing swept under the phrase "marketing purposes," telephone numbers are not mentioned explicitly either. It was necessary to deduce practices from a very careful and nuanced reading, frequently referring to multiple sections of the policy and then putting pieces together like a jigsaw puzzle. One could even make the case that answering "The policy does not say" is correct in cases as above where "information you provide" may be used for "marketing purposes" is by no means an explicit statement about telemarketing. However, we think it is important to note that the company likely does believe they have conveyed their practices: privacy policies are vetted by lawyers and are generally expected to be able to withstand a court or FTC challenge. If necessary, companies can point to the language in their policy and show that they did not violate the text by telemarketing.

We found significant differences in accuracy scores for company and format. ${ }^{6}$ We found no significant results for time based on company but format does have significant differences. Once again layered (5.7 minutes) and Privacy Finder (5.5 minutes) are an improvement over natural language (8.2 minutes) but with the caveat that layered does not do as well for accuracy.

Even though we called out D NL as particularly indirect, it falls solidly in the middle of the accuracy scores (42\%.) When participants cannot find information in layered policies, by design they should continue to the full policy for more details. In practice this appears not to happen, with a very low accuracy of $28 \%$.

Privacy Finder does support more accurate answers $(61 \%)$ even in contrast to natural language (39\%.) Privacy Finder is the only format that requires a company to disclose, yes or no,

Table 6. Percentage correct and minutes to answer for the telemarketing question.

\begin{tabular}{|l|c|c|}
\hline Policy & \% correct & Time \\
\hline A NL & $23 \%$ & 8.7 \\
A PF & $43 \%$ & 5.9 \\
\hline B NL & $41 \%$ & 6.7 \\
B PF & $67 \%$ & 5.9 \\
B Layered & $16 \%$ & 6.2 \\
\hline C NL & $42 \%$ & 9.2 \\
C PF & $68 \%$ & 5.5 \\
\hline D NL & $42 \%$ & 7.6 \\
D PF & $82 \%$ & 3.2 \\
D Layered & $33 \%$ & 5.5 \\
\hline E NL & $65 \%$ & 10.2 \\
E PF & $56 \%$ & 5.4 \\
\hline F NL & $26 \%$ & 7.1 \\
F PF & $55 \%$ & 7.4 \\
F Layered & $34 \%$ & 5.9 \\
\hline
\end{tabular}

${ }^{6}$ Accuracy scores for telemarketing are the single exception where including "Does Not Say" as a correct answer changes whether we find significance between formats. 
if they telemarket. For example, under the heading "The ways your information may be used" D PF includes "To contact you by telephone to market services or products - unless you opt-out." Again there is a lot of variation between Privacy Finder policies based on the supplemental text they provide. For example B PF, is particularly confusing by stating in free form text "While Acme does not currently support telemarketing, it is possible that in the future Acme properties may contact you by voice telephone," directly above an automatically generated statement that they may use information for telemarketing.

\section{$5 \quad$ Psychological Acceptability Results}

After completing the initial accuracy questions, participants answered a series of questions designed to elicit their emotional reactions. Participants responded on a scale from $1=$ strongly disagree to $7=$ strongly agree. Most answers hovered right around 4, which is a neutral reaction. Higher numbers are always better.

\subsection{Ease of Finding Information}

We asked four questions about how easy it was to find information. We expected responses to these questions to reflect how well participants were able to understand a particular policy, and thus be related to the accuracy questions and times. However, we found few significant results. Participants found layered easier to understand even though they were less accurate with the layered format.

- "I feel that Acme's privacy practices are explained thoroughly in the privacy policy I read" ( $\mathrm{M}=4.7$, s.d. = 1.5.) We found significant effects for company but not format. A, B, and $\mathrm{F}$ ( $\mathrm{M}=4.8$ for all $)$ scored better than $\mathrm{C}, \mathrm{D}$, and $\mathrm{E}(\mathrm{M}=4.4$ for $\mathrm{C}$ and $\mathrm{D} ; \mathrm{M}=4.5$ for $\mathrm{E}$. $)$

- "I feel confident in my understanding of what I read of Acme's privacy policy" $(\mathrm{M}=4.7$, s.d. $=1.6$. $)$ We found no significant differences between companies or formats.

- "This privacy policy was easier to understand than most policies" ( $\mathrm{M}=$ 4.5 , s.d. $=1.5$.) We found no significant differences between companies but did find significant results for formats. Layered $(M=4.8)$ scored better than natural language $(\mathrm{M}=4.4)$ or Privacy Finder $(\mathrm{M}=4.4$. $)$

- "It was hard to find information in Acme's policy" $(\mathrm{M}=3.8$, s.d. = 1.6.) We found no significant differences between companies or formats. (Note that based on the wording for this question we had to report the inverse of responses to keep higher numbers as better.)

\subsection{Trust}

If a format conveys information well but results in lack of trust of the company, it is unlikely that corporations will adopt the format. Participants trusted Privacy Finder formats slightly more than other formats. 
- "I feel secure about sharing my personal information with Acme after viewing their privacy practices" $(\mathrm{M}=4.0$, s.d $=1.7$. $)$ We found significant effects for both company and format.

- "I believe Acme will protect my personal information more than other companies" ( $\mathrm{M}=4.0$, s.d $=1.6$.) We found significant effects for both company and format.

\subsection{Enjoyment}

We asked two questions to gauge how much participants liked reading the privacy policy. If people are unwilling to read policies then improving them does not provide much benefit. We found no significant differences between formats.

- "Finding information in Acme's privacy policy was a pleasurable experience" $(\mathrm{M}=3.7$, s.d. = 1.7.) We found no significant differences between companies or formats. This was the lowest score of all eight psychological acceptability questions.

- "If all privacy policies looked just like this I would be more likely to read them" ( $\mathrm{M}=4.2$, s.d. = 1.7.) We found significant effects for format but not company.

\section{Discussion}

Our hypotheses were not fully supported and in some cases were refuted. Both layered and Privacy Finder formats did improve times to answer, but not by much, and at the expense of accuracy for layered policies. Privacy Finder policies showed modest improvement in accuracy for complex questions but no improvement for easy questions. While the accuracy scores for Privacy Finder were low in some cases, the format does represent a step forward from the status quo. Readability did not determine outcomes for natural language policies. For natural language, in some cases it appears the practices of the company were greater determinants than the words they used to describe those practices. We found few statistically significant differences in psychological acceptability.

Many researchers start from the observation that privacy policies are not usable in their current format and suggest ways to fix the problem. All of the formats were tested were unsatisfactory with a low rate of comprehension on questions that required synthesis of information. Participants did not like privacy policies of any type, and the highest mean score on the psychological acceptability questions was barely above neutral.

Privacy researchers tend to talk about policies as being uniformly bad. We expected that more readable natural language policies would have higher accuracy scores, lower times, and improved psychological acceptability than less readable policies, but that was not the case. These results could suggest that readability metrics are not a good way to differentiate between policies. This seems unlikely because the Flesch index has proven robust in many contexts 
and we do not immediately see any reason why privacy policies should be dramatically different from other types of textual analysis. It seems more likely that the range from 32 to 46 on the Flesch index is too similar to see major variations in outcome: even the most readable policies are too difficult for most people to understand and even the best policies are confusing.

Our results are robust across a variety of different policies, but our study does not concretely identify what makes a given policy comprehensible. However, we can offer three observations. First, results from the layered format suggest participants did not continue to the full policy when the information they sought was not available on the short notice. Unless it is possible to identify all of the topics users care about and summarize to one page, the layered notice effectively hides information and reduces transparency. Second, participants struggled to map concepts in the questions to the terms used in policies. It may prove fruitful to research how people internally represent privacy concepts: which terms do they currently use and which industry terms do they understand? As suggested in the Kleimann report for printed financial statements, online privacy policies may need an educational component so readers understand what it means for a site to engage in a given practice [22]. Third, the standardized formats we studied still offer policy authors quite a bit of leeway. Companies with identical practices conveyed different information, and these differences were reflected in participants' ability to understand the policies. The flexibility of the standardized formats may undermine their expected benefits to consumers.

Our study used a between subjects rather than within subjects structure. We expect that we would see larger differences, particularly in psychological acceptability, if we were to place policies side-by-side. Prior work[7] found that when participants have both the natural language and the Privacy Finder versions available, Privacy Finder fares well. If people are reading multiple companies' policies to compare them, Privacy Finder may be advantageous. However, for just understanding a single policy, we find differences between formats are not as pronounced. By only showing one policy, our study did not capture one of the potential advantages to standardized formats. Standardized formats should be more useful once readers understand where to find information. Learning effects may play a role over time when people can take greater advantage of standardized formats as they become more familiar with their layout.

At this time, we do not recommend regulating the format of online privacy policies. While we did not find substantial benefit from the standardized formats we tested, that is not an inditement of the concept of standardized formats. Early results testing a new format for privacy policies based around a nutrition label concept are encouraging [16]. Ideally, future formats will identify problems with existing approaches and attempt to improve upon what has come before. In the future, we encourage rigorous testing for new formats before their supporters encourage wide-spread adoption.

\section{References}

1. Acquisti, A., And Grossklags, J. Privacy and rationality in individual decision 
making. Security \& Privacy Magazine, IEEE 3, 1 (January-February 2005), 26-33.

2. Anton, A., Earp, J. B., Qingfeng, H., Stufflebeam, W., Bolchini, D., And JENSEN, C. Financial privacy policies and the need for standardization. IEEE Security \&5 Privacy 2, 2 (Mar-Apr 2004), 36-45.

3. Bendrath, R. Icons of privacy, May 2007. http://bendrath.blogspot.com/ 2007/05/icons-of-privacy.html Accessed 22 Feb 2009.

4. Business WiRE. European union issues guidance on privacy notices; new notices make it easier for consumers to understand, compare policies, January 2005. http: //www.tmcnet.com/usubmit/2005/jan/1104731.htm Accessed 19 May 2009.

5. Center for Information Policy Leadership. Ten steps to develop a multilayered privacy policy, 2007. www.hunton.com/files/tbl_s47Details\% 5CFileUpload265\%5C1405\%5CTen_Steps_whitepaper.pdf Accessed 12 July 2007.

6. Children's Online Privacy Protection Act of 1998 (COPPA), Public Law No. 104191, October 1998. www.cdt.org/legislation/105th/privacy/coppa.html Accessed 27 Mar 2007.

7. Cranor, L. F., Guduru, P., and Arjula, M. User interfaces for privacy agents. ACM Transactions on Computer-Human Interaction (TOCHI) (2006).

8. Egelman, S., Tsai, J., Cranor, L. F., And Acquisti, A. Timing is everything? the effects of timing and placement of online privacy indicators. In CHI 2009 (Boston, MA, USA, April 2009).

9. Federal Trade Commission. Ftc staff revises online behavioral advertising principles, February 2009. http://www.ftc.gov/opa/2009/02/behavad.shtm Accessed 15 May 2009.

10. Graber, M. A., D'Alessandro, D. M., And Johnson-West, J. Reading level of privacy policies on internet health web sites. Journal of Family Practice (July 2002).

11. U.S. Gramm-Leach-Bliley Financial Modernization Act of 1999, Public Law no. 106-102, November 1999.

12. Hochhauser, M. Lost in the fine print: Readability of financial privacy notices, July 2001. http://www.privacyrights.org/ar/GLB-Reading.htm Accessed 27 Mar 2007.

13. HuAnG, H.-J. Language-focus instruction in EFL writing : Constructing relative clauses in definition paragraphs. In 2008 International Conference on English Instruction and Assessment (2008). http://www.ccu.edu.tw/fllcccu/2008EIA/ English/C16.pdf Accessed 22 Feb 2009.

14. Jensen, C., Potts, C., And Jensen, C. Privacy practices of Internet users: Self-reports versus observed behavior. International Journal of Human-Computer Studies 63 (July 2005), 203-227.

15. KAY, M., AND TERRY, M. Textured agreements: Re-envisioning electronic consent. Technical report cs-2009-19, David R. Cheriton School of Computer Science, University of Waterloo, 2009.

16. Kelley, P. G., Bresee, J., Reeder, R. W., and Cranor, L. F. A "nutrition label" for privacy. In Symposium on Usable Privacy and Security, (SOUPS) (2009).

17. Lemos, R. MSN sites get easy-to-read privacy label. CNET News.com (2005). http://news.com.com/2100-1038_3-5611894.html Accessed 30 May 2007.

18. My Byline MediA. The Flesch reading ease readability formula. http://www . readabilityformulas.com/flesch-reading-ease-readability-formula.php Accessed 9 Mar 2009.

19. OUT-LAW NEws. Drop the jargon from privacy policies, says privacy chief, September 2005. http://www.out-law.com/page-5791 Accessed 23 Mar 2007. 
20. Pollach, I. What's wrong with online privacy policies? Communications of the ACM 30, 5 (September 2007), 103-108.

21. Reeder, R. W., Kelley, P. G., McDonald, A. M., and Cranor, L. F. A user study of the expandable grid applied to P3P privacy policy visualization. In WPES '08: Proceedings of the 7th ACM workshop on Privacy in the electronic society (2008), ACM, pp. 45-54. http://portal.acm.org/citation.cfm?id=1456403. 1456413\# Accessed 22 Feb 2009.

22. Report by Kleimann Communication Group for the FTC. Evolution of a prototype financial privacy notice, 2006. http://www.ftc.gov/privacy/ privacyinitiatives/ftcfinalreport060228.pdf Accessed 2 Mar 2007.

23. Saltzer, J. H., And Schroeder, M. D. The protection of information in computer systems. Proceedings of the IEEE 63 (September 1975), 1278-1308.

24. Sheng, X., AND CRAnOR, L. F. An evaluation of the effect of US financial privacy legislation through the analysis of privacy policies. I/S - A Journal of Law and Policy for the Information Society 2, 3 (Fall 2006), 943-980.

25. The Center for Information Policy Leadership, H. . W. L. Multi-layered notices. http://www.hunton.com/Resources/Sites/general.aspx?id=328 Accessed 23 Mar 2007.

26. The Office of the Privacy Commissioner. Release of privacy impact assessment guide and layered privacy policy, August 2006. http://www.privacy.gov . au/news/06_17.html Accessed 22 Feb 2009.

27. Tsai, J., Egelman, S., Cranor, L. F., And Acquisti, A. The effect of online privacy information on purchasing behavior: An experimental study. In The 6th Workshop on the Economics of Information Security (WEIS) (2008). http:// weis2007.econinfosec.org/papers/57.pdf Accessed 22 Feb 2009.

28. Vila, T., Greenstadt, R., And Molnar, D. Why we can't be bothered to read privacy policies: models of privacy economics as a lemons market. ACM International Conference Proceeding Series 5 (2003), 403-407.

29. W3C Working Group. The platform for privacy preferences 1.1 (P3P1.1) specification, November 2006. http://www.w3.org/TR/P3P11/ Accessed 28 Mar 2007.

\section{Appendix}

This appendix includes supporting statistical details. We performed all tests of statistical significance at the $\alpha=5 \%$ confidence level. We performed ANOVA analysis for both time data and psychological acceptability, which we recorded on a seven point Likert scale and treated as continuous variables. Accuracy questions were categorical data (either accurate or inaccurate) so we used Chi Squared tests. Details of that analysis follows.

\subsection{Accuracy}

Accuracy scores are all reported as the percentage of people who answered the question correctly. Answers are always either Yes, No, or the policy Does Not Say. We tested for statistically significant differences in mean accuracy rates by company (Table 7) and by format (Table 8). 
Table 7. Statistical Significance Tests for Accuracy Questions by Company

\begin{tabular}{|l|c|c|c|c|}
\hline Question & d.f. & $\chi^{2}$ value & $p$ & Significant? \\
\hline Cookies & 5 & 12.16 & .033 & $\checkmark$ \\
Opt Out Link & 5 & 108.31 & $<.001$ & $\checkmark$ \\
Share Email & 5 & 22.43 & $<.001$ & $\checkmark$ \\
Telemarketing & 5 & 24.99 & $<.001$ & $\checkmark$ \\
\hline
\end{tabular}

Table 8. Statistical Significance Tests for Accuracy Questions by Format

\begin{tabular}{|l|c|c|c|c|}
\hline Question & d.f. & $\chi^{2}$ value & \multicolumn{1}{|c|}{$p$} & Significant? \\
\hline Cookies & 2 & 28.95 & $<.001$ & $\checkmark$ \\
Opt Out Link & 2 & 40.80 & $<.001$ & $\checkmark$ \\
Share Email & 2 & 1.90 & .387 & \\
Telemarketing & 2 & 50.08 & $<.001$ & $\checkmark$ \\
\hline
\end{tabular}

\subsection{Time}

We recorded time in milliseconds though we reported it in minutes to assist readability. With such a fine grain unit of measure time is nearly continuous and we used ANOVA for analysis. We tested for statistically significant differences in mean times to answer by company (Table 9) and by format (Table 10).

Table 9. Statistical Significance Tests for Time to Answer by Company

\begin{tabular}{|l|c|c|c|c|}
\hline Question & d.f. & F value & $p$ & Significant? \\
\hline Cookies & 5 & 1.18 & .320 & \\
Opt Out Link & 5 & 5.58 & $<.001$ & $\checkmark$ \\
Share Email & 5 & 1.81 & .109 & \\
Telemarketing & 5 & 1.75 & .122 & \\
\hline
\end{tabular}

\subsection{Psychological Acceptability}

We asked a series of questions to capture subjective impressions of the privacy policies. Responses were on a seven point Likert scale which is sufficient granularity to treat them as continuous variables. We performed ANOVA analysis to test for statistically significant differences in mean Likert scores by company (Table 11) and by format (Table 12). 
Table 10. Statistical Significance Tests for Time to Answer by Format

\begin{tabular}{|l|c|c|c|c|}
\hline Question & d.f. & F value & \multicolumn{1}{|c|}{$p$} & Significant? \\
\hline Cookies & 2 & 4.50 & $<.012$ & $\checkmark$ \\
Opt Out Link & 2 & 3.59 & .028 & $\checkmark$ \\
Share Email & 2 & 0.15 & .864 & \\
Telemarketing & 2 & 8.59 & $<.001$ & $\checkmark$ \\
\hline
\end{tabular}

Table 11. Statistical Significance Tests for Psychological Acceptability by Company

\begin{tabular}{|l|l|c|c|c|c|}
\hline Topic & Question & d.f. & F value & $p$ & Significant? \\
\hline Finding Info. & Explained thoroughly & 5 & 1.9 & .038 & $\checkmark$ \\
Finding Info. & Confident understood & 5 & 1.9 & .099 & \\
Finding Info. & Easier to understand & 5 & 1.6 & .148 & \\
Finding Info. & Hard to find & 5 & .75 & .589 & \\
\hline Trust & Feel secure & 5 & 7.0 & $<.001$ & $\checkmark$ \\
Trust & Protect more & 5 & 3.9 & .020 & $\checkmark$ \\
\hline Enjoyment & Pleasurable & 5 & 1.7 & .135 & \\
Enjoyment & Likely to read & 5 & 2.4 & .096 & \\
\hline
\end{tabular}

Table 12. Statistical Significance Tests for Psychological Acceptability by Format

\begin{tabular}{|l|l|c|c|c|c|}
\hline Topic & Question & d.f. & F value & $p$ & Significant? \\
\hline Finding Info. & Explained thoroughly & 2 & 1.6 & .203 & \\
Finding Info. & Confident understood & 2 & .33 & .722 & \\
Finding Info. & Easier to understand & 2 & 2.89 & .051 & \\
Finding Info. & Hard to find & 2 & .60 & .549 & \\
\hline Trust & Feel secure & 2 & 14.4 & $<.001$ & $\checkmark$ \\
Trust & Protect more & 2 & 8.0 & $<.001$ & $\checkmark$ \\
\hline Enjoyment & Pleasurable & 2 & .62 & .539 & \\
Enjoyment & Likely to read & 2 & 2.4 & .032 & $\checkmark$ \\
\hline
\end{tabular}

